\title{
Skin and immune cells crosstalk via circadian regulations
}

\author{
Kanami Orihara*i] \\ School of Life Science and Technology, Tokyo Institute of Technology, 4259 Nagatsuta-cho, Midoriku, Yokohama, Kanagawa \\ 226-8501, Japan
}

*Correspondence: Kanami Orihara, School of Life Science and Technology, Tokyo Institute of Technology, 4259 Nagatsuta-cho, Midoriku, Yokohama, Kanagawa 226-8501, Japan. orihara.k.ab@m.titech.ac.jp

Academic Editor: Masutaka Furue, Kyushu University, Japan

Received: July 21, 2021 Accepted: September 13, 2021 Published: October 31, 2021

Cite this article: Orihara K. Skin and immune cells crosstalk via circadian regulations. Explor Immunol. 2021;1:285-94. https:// doi.org/10.37349/ei.2021.00019

\begin{abstract}
Both innate and adaptive immune cells exist in the skin, predominantly in the dermis layer. Recent studies have focused on how and which circadian rhythms contribute to maintain good health. Over recent years, we have gained a better understanding of the molecular mechanisms that control biological clocks and circadian rhythms. Circadian rhythms maintain homeostasis by providing day and night information to various physiological functions of our body. However, excessively high immune system activity can lead to a risk of developing autoimmune or allergic diseases. Recently, increasing numbers of studies with human and mouse models have been conducted to investigate the mechanisms underlying circadian regulation of the skin homeostasis. In this review, circadian regulation in the skin will be discussed from different points of view. Skin is referred as the largest organ of the body and is directly exposed to the external environment, including large changes in diurnal temperature, light, and pathogens. Immune cells as well as skin cells are the ones protecting us from these stimulants. Associations of the circadian system and these cells have been revealed in many ways, however, the specific roles of the peripheral clocks in these cells remain unknown. Circadian regulation in the skin diseases is discussed specifically in atopic dermatitis and other skin allergic symptoms as well as psoriasis.
\end{abstract}

\section{Keywords}

Skin, immune cells, immunity, chronobiology, circadian rhythm, body clock, homeostasis

\section{Introduction}

Most living things maintain an approximate $24 \mathrm{~h}$ rhythm in behaviors, including sleep-wake cycles and physiological processes such as body temperature and hormone secretion [1]. These are referred to as circadian rhythms and are controlled by clocks, which introduce rhythms via an internal factor without the need for participation of environmental factors, although rhythms can be adjusted by external factors [2]. Circadian rhythms maintain homeostasis by providing day and night information to various physiological functions of our body. In other words, circadian rhythms have an influence on a wide variety of biological processes, including behavior, metabolism, neuronal activity, endocrinology, and immunity [2]. For instance,

(C) The Author(s) 2021. This is an Open Access article licensed under a Creative Commons Attribution 4.0 International License (https://creativecommons.org/licenses/by/4.0/), which permits unrestricted use, sharing, adaptation, distribution and reproduction in any medium or format, for any purpose, even commercially, as long as you give appropriate credit to the original author(s) and the source, provide a link to the Creative Commons license, and indicate if changes were made. 
the peripheral clock in cell level is shown to operate pigmentation in human hair follicle as well as in epidermal melanocytes [3].

In humans, circadian rhythms are generated in and coordinated by a multi-oscillatory system located in the brain [4]. This master pacemaker is located in the suprachiasmatic nucleus (SCN) of the hypothalamus. The SCN shows a strong $24 \mathrm{~h}$ cycle rhythm of electrophysiological activity, which leads to periodic display of physiological functions and behavior via a hierarchical system $[2,4]$. Thus, the SCN is referred to as the central clock, and other clocks located throughout the body are known as peripheral clocks [2]. The human body is said to be composed of approximately 37 trillion cells, in almost all of which peripheral clocks contribute to maintenance of functions in peripheral tissue [2].

Over recent years, we have gained a better understanding of the molecular mechanisms that control biological clocks and circadian rhythms. Indeed, many studies have supported the importance of molecular clocks for the regulation of human physiology, including hormonal and metabolic homeostasis. Studies in fruit flies, and subsequently in rodents and humans, have led to the development of a transcriptionaltranslational feedback loop (TTFL) molecular model of circadian rhythms. The major clock genes involved in the TTFL include brain and muscle aryl hydrocarbon receptor nuclear translocator-like 1 (BMAL1), circadian locomotor output cycles kaput (CLOCK), period circadian regulator (PER)1/2, and cryptochrome circadian regulator $(C R Y) 1 / 2$, which form the main components of systems with a $24 \mathrm{~h}$ cycle. The transcriptional activators BMAL1 and CLOCK play a positive role by activating PER and $C R Y$ genes via specific promoter sequences known as E-boxes. PER and $C R Y$ are then translated into proteins in the cytoplasm and transported to the nucleus after interacting with each other. Transcription is then stop when they bind to BMAL1 and CLOCK. Thus, PER and CRY are expressed rhythmically in a $24 \mathrm{~h}$ cycle [5].

In humans, the strongest environmental factor that acts to reset the central clock is sunlight or visible light acting through the retina, and subsequently triggering hormonal and other electrochemical signals in the body [4]. In chronobiology, this process is known as entrainment, which refers to the synchronization of physiological or behavioral rhythms to environmental rhythms. Peripheral organs and cells are functionally entrained by hormones and other electrochemical signals, or by body temperature [4]. One of the key features of circadian rhythms is that the circadian clock maintains an endogenous periodic rhythm of approximately $24 \mathrm{~h}$, even in constant dark conditions or in the absence of any environmental stimulation [6]. Therefore, light and temperature work synergistically to orchestrate the circadian phase, modulating regulation of the clock and contributing to seasonal adaptations in clock functions. Moreover, recent studies have focused on how nutrition ("eating right") might affect circadian rhythms to maintain good health [7].

While the central clock is primarily responsible for light entering the retina and stimulating the SCN via the hypothalamic retinal tract, the skin, which deserves to be the mid-wall between the environment and the organism, receives and generates signals regarding timing. Since the skin is directly exposed to the external environment, skin functions, including immune responses, are influenced by the central clock but can be locally reset by exposure to ultraviolet (UV) light, pollutants, humidity, temperature, etc. [4]. Therefore, the influence of skin peripheral clock on the skin immunity may be closely related to the circadian system and the maintenance of healthy skin at both cellular and molecular levels.

\section{Circadian regulation in the skin}

Skin is regarded as the largest organ of the body and is directly exposed to the external environment, including large changes in diurnal temperature, light, and pathogens. Thus, in normal circumstances, many markers of human skin moisture retention, transepidermal water loss (TEWL), capillary blood flow, temperature and $\mathrm{pH}$ of the skin surface, and rate of epithelial cell proliferation appear to maintain daily rhythms to keep our skin healthy [4]. TEWL is higher in the afternoon and the evening than in the morning [4]. This suggests that performance of the skin as a barrier declines in the evening and at night. Skin temperature of the arm and calf are also reported to show daily variations, with the lowest temperatures in early morning and the highest at night [8]. Skin temperature typically reflects cutaneous capillary flow, which also shows a pattern of being lower in the morning and the highest in the afternoon, with a second peak in the late evening just before sleep [9]. Moreover, patients with chronic urticaria 
suffer the greatest itchiness in the evening [10], which can be explained by evidence for a decreased skin barrier in the evening. Mizutani et al. [11] reported that although cutaneous tolerance was induced in mice, with sensitization during the neonatal period, this induction was suppressed in Bmall-deficient mice, mice with dysregulated circadian rhythms, suggesting that circadian control regulates homeostasis of the skin. Looking back in some old studies, in 1994, Pershing et al. [9] have reported circadian activity of topical $0.05 \%$ betamethasone dipropionate in human skin in vivo. Although the precise mechanisms were not discussed in this study, the authors suggest an application of topical treatment on the skin at late afternoon may be more effective than the same amount of treatment in the morning [9].

In 2001, circadian rhythms in heat-induced sweat on forearm were shown. Skin surface pH shows fluctuation within the range of $\mathrm{pH} 5.5$ to 6.0 , and $\mathrm{pH}$ was found to be higher in the afternoon compared to the morning [12]. There are also other evidence shown about circadian changes in the volume of heatinduced [13] or exercise-induced [14] sweat. Since sweat is a sample limited in the volume, there are still not many studies following these suggestions. However, as it is also a sample that can be collected invasively, any further studies are highly expected.

Living organisms are active at different periods (diurnal or nocturnal) depending on the species. During the active period, pathogens are often encountered. The $24 \mathrm{~h}$ cyclical control of immune function enables organisms to protect themselves with the most effective response when it is needed. Another role of circadian rhythms is to adjust various immune functions (e.g., among different cell types) without any delay in cooperation with other physiological systems [15]. However, excessively high immune system activity can lead to a risk of developing autoimmune or allergic diseases. Thus, circadian regulation in the skin aims to maintain a homeostasis within the skin and the immune system.

\section{Clock-related molecules in skin cells}

An in vitro assessment of keratinocytes, fibroblasts, and adipocytes has clarified contributions of the cellular circadian clocks in these cells to cutaneous homeostasis [16]. Moreover, Bmal1 deficient mice were reported to show fast aging in cutaneous cells [17], which may also contribute to a premature immune system for regulating the skin condition. However, studies investigating the precise pathophysiology of the cutaneous tissue and the skin are yet to be conducted.

Although the numbers are limited, there are several important studies investigating the presence of circadian oscillations in the human skin have reported. Sandu et al. [18] reported that primary cultures of keratinocytes, melanocytes, and fibroblasts derived from a human skin biopsy show circadian oscillations in clock related genes. The analysis also revealed with each cell type specific oscillating periods, which fibroblasts showed the shortest period of $22.2 \mathrm{~h}$, followed by keratinocytes $23.1 \mathrm{~h}$, and melanocytes $25.2 \mathrm{~h}$. They also detected opposite phases between Bmal1, and Per or Cry transcripts, approximately 8 h phase advance in Per [18], in accordance with the TTFL theory.

\section{Clock-related pathways in skin}

In 2014, Hardman et al. [3] also showed evidence in melanocytes as well as hair follicles, in which they investigated a cell-autonomous pigmentation modulatory function in peripheral clocks. Using siRNA technology, BMAL1 or PER1 silencing was found to increase hair pigmentation. These partial clock gene silencing resulted in to increase tyrosinase and tyrosinase related protein (TRP)1/2 expression and activity, as well as melanin synthesis [3].

Another study showed Krueppel-like factor 9 (Klf9), a transcriptional factor known to be involved in cell proliferation and differentiation including keratinocytes, showed a clear diurnal oscillation mRNA expression in epidermal suction blister and punch-biopsy samples [19]. Similarly, Bmal1 protein was found to play a role in regulating cell proliferation and metabolism in mouse skin via controlling anti-phasic parameter, reactive oxygen species (ROS) levels [20]. Although the study was based on fibroblasts data, ROS as well as UV stimulation is shown to trigger the synchronization of the circadian clock via interactions between BMAL1 and heat-shock factor-1 (HSF1), a central transcription factor for the heat-shock response (HSR) pathway [21]. 
These data support ROS contribution to the circadian regulation in the skin. Geyfman et al. [20] also reported another role of Bmal1 adjusting circadian sensitivity to ultraviolet-B (UV-B) induced DNA damage in mouse epidermis; sensitivity is higher late at night. This finding is in consistent with the study showing UV-Binduced DNA damage responses and apoptosis in human keratinocytes are regulated by CLOCK as well as BMAL1 proteins [22].

\section{Immune factors regulated by clock-related molecules}

Regarding UV-B damage, tissue inhibitor of metalloproteinase-3 (TIMP-3), which is regulated by CLOCK, was shown as a novel circadian locomotor that inhibits the expression of UV-B-induced matrix metallopeptidase 1(MMP1) and inflammatory cytokines [tumor necrosis factor (TNF), chemokine (C-X-Cmotif) ligand (CXCL)1, CXCL8] in human keratinocytes [23]. CLOCK as well as BMAL1 is also reported to regulate the transcription of aquaporin-3 (AQP3), which $24 \mathrm{~h}$ cycle rhythmical expression pattern was detected in HaCaT cells. AQP3 locates in the basal layer of the epidermis and regulates biological functions of the skin such as water content and trans-epidermal water loss, thus this study revealed a molecular mechanism to control the skin hydration [24]. These data suggest that skin circadian protein oscillations are essential for the skin, as an outermost shield that protects our body from harmful and complicated external conditions such as UV exposures and dryness.

Considering the shield barrier function of the skin, antimicrobial peptides are also shown to oscillate daily. In this study, the mRNA expression levels in antimicrobial peptides analyzed, including defensin 1 and 3 , reached their peaks at early hours of active phase, rhythms if which were abolished in mice kept in constant dark condition [25]. This study suggests that the skin innate immune response is influenced by the circadian system, suggesting that we may make use of the circadian influence to control the skin immunity.

As was mentioned earlier, "eating right" is the focus of attention recently in order to keep peripheral clocks to maintain our good health. Using 5 different patterns of restricted feeding in mice, Wang et al. [26] showed skin peripheral clock shift can be induced by restricted feeding, independent from liver clock. Moreover, restriction during daytime was reported to reverse the UV sensitivity of the skin, especially for DNA damage. Although the direct interconnection between the feeding signal and the skin peripheral clock is still unknown, influence of food intake on the skin biophysiology may be greater than it is recognized so far (Figure 1).

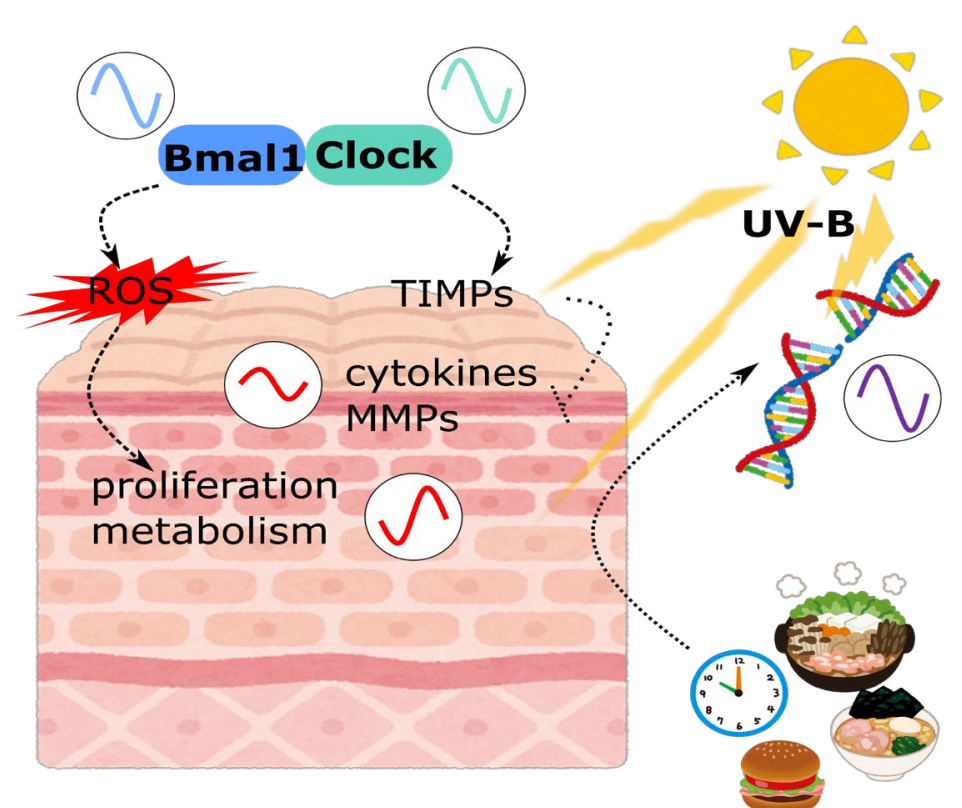

Figure 1. Circadian regulation in skin cells. Circadian rhythms in skin are affected by many factors. Not only clock related molecules, but other skin physiologies are also regulated by these signals 


\section{Circadian regulation in immune cells}

Both innate and adaptive immune cells exist in the skin, predominantly in the dermis layer. The epidermis, especially the basal layer, contains Langerhans cells (LCs) and CD8 ${ }^{+} \mathrm{T}$ cells. On the other hand, the dermal layer is composed of $\mathrm{CD}^{+} \mathrm{T}$ cells, B cells, natural killer (NK) cells, innate lymphoid cells, eosinophils, neutrophils, mast cells, macrophages, and dendritic cells [27]. LC initiates the immune response in the skin; they form a network on the surface of the skin, extends the dendrites, and capture antigens in the environment through the gaps of tight junctions. Upon phagocytosing an allergen or pathogen, LC presents the antigen directly to $\mathrm{T}$ cells present in cutaneous immunological memory, or migrates to the lymph nodes and presents the antigen via major histocompatibility complex (MHC) [28].

Circadian clock genes are expressed in numerous immunological tissues and cells, and are reported to show rhythmic expression in spleen, lymph node, and peritoneal macrophages, tissues, and cells [29]. The manner by which physiology is controlled by peripheral clocks is still poorly understood. However, a $24 \mathrm{~h}$ cycle does seem to be important for the skin homeostasis, as has been observed in sleep disruptions in many of the skin disorders. Additionally, many symptoms of allergic diseases and associated clinical laboratory data are reported to show marked changes across approximately $24 \mathrm{~h}$ cycles [30]. Moreover, these circadian clocks can be operated autonomously without signals from the central clock [29].

It is well known that both endogenous (secreted from adrenal glands) and exogenous (therapeutic) glucocorticoid hormones modulate peripheral clocks [31]. Cortisol, an endogenous glucocorticoid hormone in humans, is reported to show $24 \mathrm{~h}$ cycle variations, reaching a peak at the time of waking and a trough late at night [32]. In conditions without any specific stress, plasma corticosterone levels in mice show 5 to 10 fluctuations between the highest and lowest concentrations [33]. Among currently available medical options, topical glucocorticoids are widely used to treat allergic symptoms, different classes of glucocorticoid ointments are used for atopic dermatitis/eczema. Therefore, understanding the role of glucocorticoids on local clocks is important to determine the regulation of circadian rhythms on the immune system. Moreover, it will enable more accurate and personalized treatment of patients.

The role of circadian rhythms in the immune system is a new area of research, which has started to reveal the importance of circadian control in immune regulation. However, the specific underlying mechanisms remain unknown. Research generally supports the role of circadian-controlled immune variations in coping with infections [34]. For instance, the circulating leukocyte count reaches a peak during the day in rodents and at night in humans [16]. Moreover, the concentration of human serum interleukin (IL)- 5 appears to be maintained by type 2 innate lymphoid cells (ILC2), which constitutively secrete IL-5. They also secrete IL-13 during type 2 inflammation, which leads to local CC chemokine ligand (CCL)11/eotaxin production and eosinophil accumulation as observed in many allergic symptoms [35]. This indicates that not all variables in the immune system follow patterns modulated by circadian rhythms, but some do so to keep the homeostasis in the immune system.

In rodents, Clock mutant mice have been well studied in the chronobiology field. One study reported that, compared with wild type (WT) mice, Clock mutant mice showed comparable numbers and proportions of lymphocytes (CD4 ${ }^{+}, \mathrm{CD}^{+}, \gamma / \delta^{+} \mathrm{T}$ cells, and B cells) in the thymus and spleen, as well as serum antibody [immunoglobulin (Ig)G, IgA, and IgE] levels, and leukocyte counts in peripheral blood. Thus, Clock mutant mice do not have obvious developmental defects in terms of the immune system [36]. This suggests that CLOCK may not have a direct impact on immune regulation. Nevertheless, impaired leukocyte trafficking and the skin inflammatory responses was shown in hamsters lacking a functional circadian system by disruptive phase shifts [37], which suggests a solid circadian regulation in the skin immune system. Also, in human study, the numbers and suppressive function of $\mathrm{CD} 4^{+} \mathrm{CD} 25^{+} \mathrm{Foxp}^{+}$regulatory $\mathrm{T}$ cells (Treg) was reported to show a circadian rhythm, increase at night and decrease during the day [11]. Taken together, although some of the precise mechanism is still unveiled yet, the circadian clock may be enhancing the skin immunity during active periods when the risk of contracting infections is the highest, and suppressing immune responses during inactive periods. 


\section{Circadian regulation in atopic dermatitis and other skin allergic symptoms}

Sleep disruption in skin disorders has been described in many studies. In addition to the diurnal cortisol pattern affecting the night increased itching in atopic dermatitis, urticaria, and several other skin diseases [10,38], elevated TEWL in the evening seems to be related to elevated pruritus [38]. Circadian clock dysregulation was also reported to affect contact hypersensitivity. Clock mutant mice showed significantly severe ear swelling compared to WT mice [39]. They also found significantly higher numbers of mast cells in Clock mutant model [39]. Per2 is also reported to regulate a time-of-day-dependent variation in cutaneous anaphylactic reaction [40].

Mizutani et al. [11] reported that cutaneous tolerance was induced in mice with sensitization during the neonatal period. Those induction was suppressed in mice with dysregulated circadian rhythm induced in two different methods [11], suggesting circadian control regulates the homeostasis in the skin. Also, a high amplitude in internal cortisol level was reported to decrease the immune function against Staphylococcus aureus colonization on mice skin, which is an exacerbating factor in atopic dermatitis [41]. Interestingly, a study with 2,4-dinitrofluorobenzene (DNFB)-treated NC/Nga mice showed that increased ear thickness and the skin lesions can be reduced by topical melatonin administration [42]. Moreover, total IgE levels as well as IL-4 and interferon (IFN)- $\gamma$ production from local CD $4^{+} \mathrm{T}$ cells were decreased by the melatonin treatment [42]. Melatonin is a hormone that plays a major role in regulation of the circadian system, which is also known to have anti-oxidant properties, as a direct free radical scavenger, by stimulating anti-oxidative enzymes to increase the efficiency of mitochondrial oxidative phosphorylation, and by reducing free radical generation [43]. These anti-oxidative functions of melatonin are suggested to influence the immune system. Exogenous melatonin treatment of pinealectomized rats was found to restore the ability of cells to migrate from the bone marrow [44]. Another study showed an activation with retinoic acid-related orphan receptor (ROR) $\alpha / \gamma$ agonist (SR1078) increased mRNA expression levels of clock related genes and worsen the atopic dermatitis-like symptoms [45]. Taken together, these data strongly support an association between the atopic dermatitis or other skin allergic conditions and the circadian system. It seems that larger amplitude in corticosteroids or clock related genes seem to lead to an exacerbation in these conditions, thus, keeping fluctuation small but enough to maintain immune function to beat back $S$. aureus is important. In order to make this happen, topical melatonin or any alternative administration may become a possible candidate.

\section{Circadian regulation in psoriasis}

In psoriasis, daily as well as seasonal variations in its symptoms has reported. In this cross-sectional online survey study, the authors showed that pruritus and flare-ups in psoriasis fluctuate daily, the worst in the evening followed by nighttime [46].

Recently, increasing numbers of studies in mouse models have been conducted to investigate the mechanisms underlying circadian regulation of the skin homeostasis. Using a Toll-like receptor 7 ligand, imiquimod (IMQ) induced psoriasis-like skin disease model, Ando et al. [36] demonstrated milder skin symptom in Clock mutant mice. The authors also clarified that Clock mutation reduced $\gamma / \delta^{+} \mathrm{T}$ cell responses to IL-23 significantly, caused by a diminished expression of IL-23R [36]. Moreover, Per2 mutation was found to exacerbate IMQ induced dermatitis [36].

Similarly, mice treated with $1 \%$ IMQ cream for 5 days showed disruption in an otherwise observed diurnal trend in epidermal thickness in homeostasis [47]. Moreover, the authors reported a constant increased spleen weight in 5 days IMQ treated mice, although the weight showed a daily fluctuation under homeostasis [47]. This suggests systemic inflammation level is elevated by IMQ treatment, i.e., in psoriasis-like skin disease condition. They also showed mice lacking Bmal1 significantly increased IMQ-induced IFN-sensitive gene response in the skin [47], which confirms the circadian regulation on IMQ-induced skin inflammation.

Although many of the mechanism still unclear, these evidences support psoriasis symptoms are influenced by the circadian regulation through both the skin and the immune cell systems. 


\section{Conclusion}

In this review, circadian regulation in the skin is shown from different points of view. Associations of the circadian system and the skin cells and the immune cells have been revealed in many ways, however, specific roles of the peripheral clocks in the immune cells as well as epithelial cells and mesenchymal cells remain unknown. Especially, how the peripheral clocks are dysregulated in disease status in cellular or tissue level assessments are required to clarify the complicated network web of these systems. Harmonizing the advanced studies from both immunology and chronobiology viewpoints, namely in chrono-immunology, may positively influence our future health, diagnosis, therapies, and prevention. Medically or cosmetically, we may apply topical treatment to reset local clocks, which may lead to keeping our skin healthy and promote a better and a sooner recovery in near future (Figure 2).

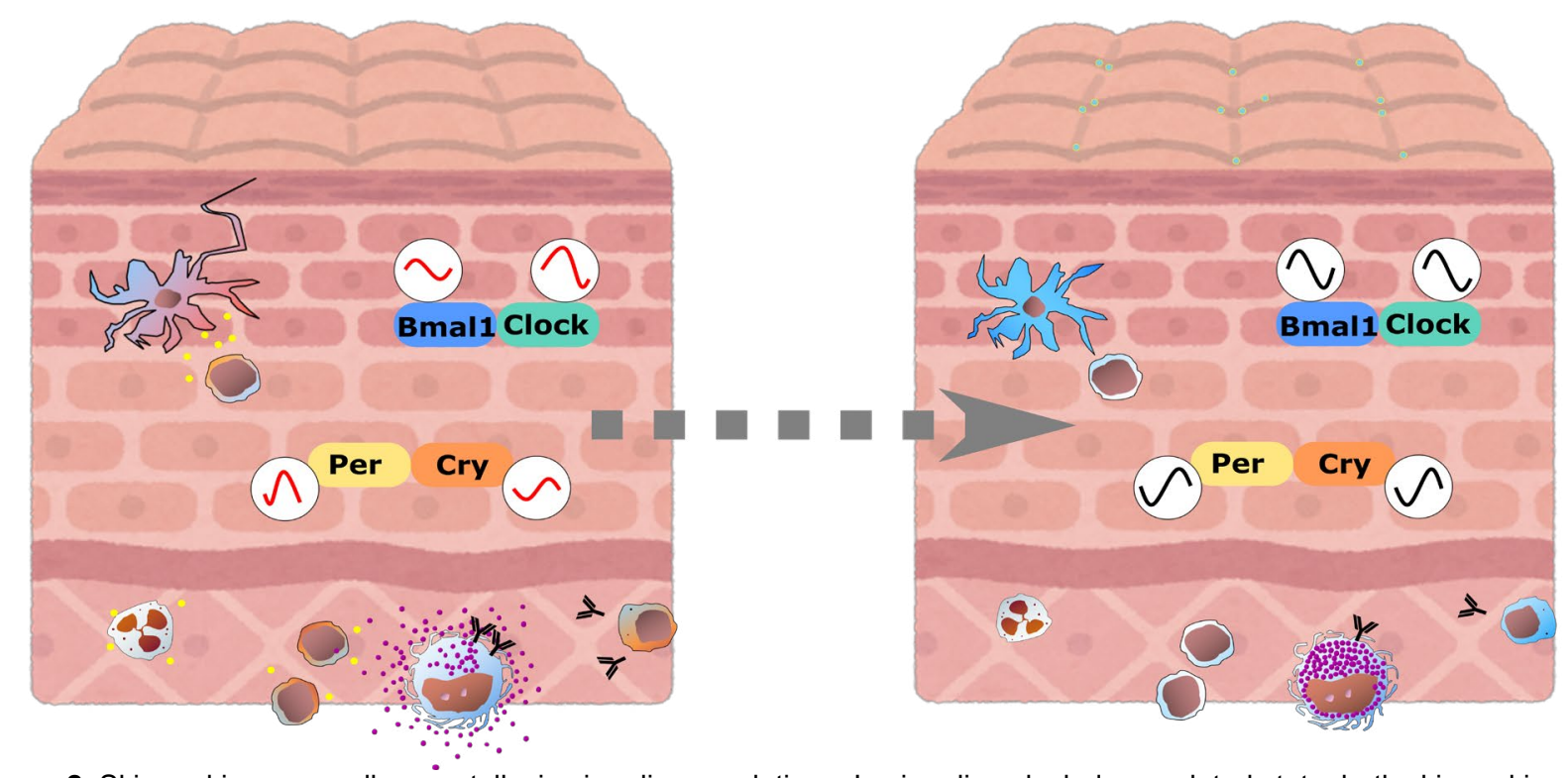

Figure 2. Skin and immune cells crosstalk via circadian regulations. In circadian clock dysregulated state, both skin and immune cells interact and function abnormally, expanding inflammation. Upon resetting local clock, cells may be brought back to steady state and will get ready highly for protecting themselves

\section{Abbreviations}

BMAL1: brain and muscle aryl hydrocarbon receptor nuclear translocator-like 1

CLOCK: circadian locomotor output cycles kaput

CRY: cryptochrome circadian regulator

Ig: immunoglobulin

IL: interleukin

IMQ: imiquimod

LCs: Langerhans cells

PER: period circadian regulator

ROS: reactive oxygen species

SCN: suprachiasmatic nucleus

TEWL: transepidermal water loss

TTFL: transcriptional-translational feedback loop

UV: ultraviolet

UV-B: ultraviolet-B 


\section{Declarations}

Author contributions

The author contributed solely to the work.

\section{Conflicts of interest}

The author declares that there are no conflicts of interest.

\section{Ethical approval}

Not applicable.

\section{Consent to participate}

Not applicable.

\section{Consent to publication}

Not applicable.

\section{Availability of data and materials}

Not applicable.

Funding

Not applicable.

\section{Copyright}

(C) The Author(s) 2021.

\section{References}

1. Tanabe K, Kitagawa E, Wada M, Haraguchi A, Orihara K, Tahara Y, et al. Antigen exposure in the late light period induces severe symptoms of food allergy in an OVA-allergic mouse model. Sci Rep. 2015;5:14424.

2. Johnston JD, Ordovás JM, Scheer FA, Turek FW. Circadian rhythms, metabolism, and chrononutrition in rodents and humans. Adv Nutr. 2016;7:399-406.

3. Hardman JA, Tobin DJ, Haslam IS, Farjo N, Farjo B, Al-Nuaimi Y, et al. The peripheral clock regulates human pigmentation. J Invest Dermatol. 2015;135:1053-64.

4. Matsui MS, Pelle E, Dong K, Pernodet N. Biological rhythms in the skin. Int J Mol Sci. 2016;17:801.

5. Orihara K, Haraguchi A, Shibata S. Crosstalk among circadian rhythm, obesity and allergy. Int J Mol Sci. 2020;21:1884.

6. Tahara Y, Shibata S. Chrono-biology, chrono-pharmacology, and chrono-nutrition. J Pharmacol Sci. 2014;124:320-35.

7. Tahara Y, Shibata S. Entrainment of the mouse circadian clock: effects of stress, exercise, and nutrition. Free Radic Biol Med. 2017;119:129-38.

8. McDonnell AC, Eiken O, Mekjavic PJ, Mekjavic IB. Circadian rhythm of peripheral perfusion during 10-day hypoxic confinement and bed rest. Eur J Appl Physiol. 2014;114:2093-104.

9. Pershing LK, Corlett JL, Lambert LD, Poncelet CE. Circadian activity of topical $0.05 \%$ betamethasone dipropionate in human skin in vivo. J Invest Dermatol. 1994;102:734-9.

10. Maurer M, Ortonne JP, Zuberbier T. Chronic urticaria: an internet survey of health behaviours, symptom patterns and treatment needs in European adult patients. Br J Dermatol. 2009;160:633-41.

11. Mizutani H, Tamagawa-Mineoka R, Minami Y, Yagita K, Katoh N. Constant light exposure impairs immune tolerance development in mice. J Dermatol Sci. 2017;86:63-70. 
12. Burry J, Coulson HF, Roberts G. Circadian rhythms in axillary skin surface pH. Int J Cosmet Sci. 2001;23:207-10.

13. Aoki K, Kondo N, Shibasaki M, Takano S, Tominaga H, Katsuura T. Circadian variation of sweating responses to passive heat stress. Acta Physiol Scand. 1997;161:397-402.

14. Waterhouse J, Aizawa S, Nevill A, Edwards B, Weinert D, Atkinson G, et al. Rectal temperature, distal sweat rate, and forearm blood flow following mild exercise at two phases of the circadian cycle. Chronobiol Int. 2007;24:63-85.

15. Labrecque N, Cermakian N. Circadian clocks in the immune system. J Biol Rhythms. 2015;30:277-90.

16. Plikus MV, Van Spyk EN, Pham K, Geyfman M, Kumar V, Takahashi JS, et al. The circadian clock in skin: implications for adult stem cells, tissue regeneration, cancer, aging, and immunity. J Biol Rhythms. 2015;30:163-82.

17. Kowalska E, Ripperger JA, Hoegger DC, Bruegger P, Buch T, Birchler T, et al. NONO couples the circadian clock to the cell cycle. Proc Natl Acad Sci U S A. 2013;110:1592-9.

18. Sandu C, Dumas M, Malan A, Sambakhe D, Marteau C, Nizard C, etal. Human skin keratinocytes, melanocytes, and fibroblasts contain distinct circadian clock machineries. Cell Mol Life Sci. 2012;69:3329-39.

19. Spörl F, Korge S, Jürchott K, Wunderskirchner M, Schellenberg K, Heins S, et al. Krüppel-like factor 9 is a circadian transcription factor in human epidermis that controls proliferation of keratinocytes. Proc Natl Acad Sci U S A. 2012;109:10903-8.

20. Geyfman M, Kumar V, Liu Q, Ruiz R, Gordon W, Espitia F, etal. Brain and muscle Arnt-like protein-1 (BMAL1) controls circadian cell proliferation and susceptibility to UVB-induced DNA damage in the epidermis. Proc Natl Acad Sci U S A. 2012;109:11758-63.

21. Kawamura G, Hattori M, Takamatsu K, Tsukada T, Ninomiya Y, Benjamin I, et al. Cooperative interaction among BMAL1, HSF1, and p53 protects mammalian cells from UV stress. Commun Biol. 2018;1:204.

22. Sun Y, Wang P, Li H, Dai J. BMAL1 and CLOCK proteins in regulating UVB-induced apoptosis and DNA damage responses in human keratinocytes. J Cell Physiol. 2018;233:9563-74.

23. Park S, Kim K, Bae IH, Lee SH, Jung J, Lee TR, et al. TIMP3 is a CLOCK-dependent diurnal gene that inhibits the expression of UVB-induced inflammatory cytokines in human keratinocytes. FASEB J. 2018;32:1510-23.

24. Matsunaga N, Itcho K, Hamamura K, Ikeda E, Ikeyama H, Furuichi Y, et al. 24-hour rhythm of aquaporin-3 function in the epidermis is regulated by molecular clocks. J Invest Dermatol. 2014;134:1636-44.

25. Bilska B, Zegar A, Slominski AT, Kleszczynski K, Cichy J, Pyza E. Expression of antimicrobial peptide genes oscillates along day/night rhythm protecting mice skin from bacteria. Exp Dermatol. 2021;30:1418-27.

26. Wang H, van Spyk E, Liu Q, Geyfman M, Salmans ML, Kumar V, et al. Time-restricted feeding shifts the skin circadian clock and alters UVB-induced DNA damage. Cell Rep. 2017;20:1061-72.

27. Nguyen AV, Soulika AM. The dynamics of the skin's immune system. Int J Mol Sci. 2019;20:1811.

28. Chambers ES, Vukmanovic-Stejic M. Skin barrier immunity and ageing. Immunology. 2020;160:116-25.

29. Keller M, Mazuch J, Abraham U, Eom GD, Herzog ED, Volk HD, et al. A circadian clock in macrophages controls inflammatory immune responses. Proc Natl Acad Sci U S A. 2009;106:21407-12.

30. Nakao A, Nakamura Y, Shibata S. The circadian clock functions as a potent regulator of allergic reaction. Allergy. 2015;70:467-73.

31. Chung S, Son GH, Kim K. Circadian rhythm of adrenal glucocorticoid: its regulation and clinical implications. Biochim Biophys Acta. 2011;1812:581-91.

32. Fei GH, Liu RY, Zhang ZH, Zhou JN. Alterations in circadian rhythms of melatonin and cortisol in patients with bronchial asthma. Acta Pharmacol Sin. 2004;25:651-6.

33. Oster H, Damerow S, Kiessling S, Jakubcakova V, Abraham D, Tian J, et al. The circadian rhythm of glucocorticoids is regulated by a gating mechanism residing in the adrenal cortical clock. Cell Metab. 2006;4:163-73. 
34. Scheiermann C, Kunisaki Y, Frenette PS. Circadian control of the immune system. Nat Rev Immunol. 2013;13:190-8.

35. Nussbaum JC, Van Dyken SJ, von Moltke J, Cheng LE, Mohapatra A, Molofsky AB, et al. Type 2 innate lymphoid cells control eosinophil homeostasis. Nature. 2013;502:245-8.

36. Ando N, Nakamura Y, Aoki R, Ishimaru K, Ogawa H, Okumura K, et al. Circadian gene Clock regulates psoriasis-like skin inflammation in mice. J Invest Dermatol. 2015;135:3001-8.

37. Prendergast BJ, Cable EJ, Patel PN, Pyter LM, Onishi KG, Stevenson TJ, et al. Impaired leukocyte trafficking and skin inflammatory responses in hamsters lacking a functional circadian system. Brain Behav Immun. 2013;32:94-104.

38. Gupta MA, Gupta AK. Sleep-wake disorders and dermatology. Clin Dermatol. 2013;31:118-26.

39. Takita E, Yokota S, Tahara Y, Hirao A, Aoki N, Nakamura Y, et al. Biological clock dysfunction exacerbates contact hypersensitivity in mice. Br J Dermatol. 2013;168:39-46.

40. Nakamura Y, Harama D, Shimokawa N, Hara M, Suzuki R, Tahara Y, et al. Circadian clock gene Period2 regulates a time-of-day-dependent variation in cutaneous anaphylactic reaction. J Allergy Clin Immunol. 2011;127:1038-45.e1-3.

41. Hashimoto Y, Kaneda Y, Akashi T, Arai I, Nakaike S. Persistence of Staphylococcus aureus colonization on the skin of NC/Nga mice. J Dermatol Sci. 2004;35:143-50.

42. Kim TH, Jung JA, Kim GD, Jang AH, Ahn HJ, Park YS, et al. Melatonin inhibits the development of 2,4-dinitrofluorobenzene-induced atopic dermatitis-like skin lesions in NC/Nga mice. J Pineal Res. 2009;47:324-9.

43. Reiter RJ, Tan DX, Mayo JC, Sainz RM, Leon J, Czarnocki Z. Melatonin as an antioxidant: biochemical mechanisms and pathophysiological implications in humans. Acta Biochim Pol. 2003;50:1129-46.

44. Martins E Jr., de Oliveira APL, de Araujo AMF, de Lima WT, Cipolla-Neto J, Costa Rosa LF. Melatonin modulates allergic lung inflammation. J Pineal Res. 2001;31:363-9.

45. Hiramoto K, Orita K, Yamate Y, Kasahara E, Yokoyama S, Sato EF. The clock genes are involved in the deterioration of atopic dermatitis after day-and-night reversed physical stress in NC/Nga mice. Open Biochem J. 2018;12:87-102.

46. Ferguson FJ, Lada G, Hunter HJA, Bundy C, Henry AL, Griffiths CEM, et al. Diurnal and seasonal variation in psoriasis symptoms. J Eur Acad Dermatol Venereol. 2021;35:e45-7.

47. Greenberg EN, Marshall ME, Jin S, Venkatesh S, Dragan M, Tsoi LC, et al. Circadian control of interferonsensitive gene expression in murine skin. Proc Natl Acad Sci U S A. 2020;117:5761-71. 